\title{
Detection and Mitigation of JIT-Induced Side Channels ${ }^{*}$
}

\author{
Tegan Brennan \\ University of California Santa Barbara \\ Santa Barbara, CA, USA \\ tegan@cs.ucsb.edu
}

ACM Reference Format:

Tegan Brennan. 2020. Detection and Mitigation of JIT-Induced Side Channels. In 42nd International Conference on Software Engineering Companion (ICSE '20 Companion), May 23-29, 2020, Seoul, Republic of Korea. ACM, New York, NY, USA, 3 pages. https://doi.org/10.1145/3377812.3382174

\section{SIDE CHANNELS IN SOFTWARE}

Cyber-attacks stealing confidential information are becoming increasingly frequent and devastating as modern software systems store and manipulate greater amounts of sensitive data. Leaking information about private user data, such as the financial and medical records of individuals, trade secrets of companies and military secrets of states can have drastic consequences. Confidentiality of such private data is critical for users of these systems. Many software development practices, such as the encryption of packages sent over a network, aim to protect the confidentiality of private data by ensuring that an observer is unable to learn anything meaningful about a program's secret input from its public output. Under these protections, the software system's main communication channels, such as the content of the network packets it sends, or the output it writes to a public file, should not leak information about the private data. However, many software systems still contain serious security vulnerabilities. Side channels are an important class of information leaks where secret information can be captured through the observation of non-functional side effects of software systems. Potential side channels include those in execution time, memory usage, size and timings of network packets, and power consumption. Although side-channel vulnerabilities due to hardware (such as vulnerabilities that exploit the cache behavior) have been extensively studied $[1,2,10,13,15-17,19,23]$, software side channels have only recently become an active area of research, including recent results on software side-channel detection $[4,8,11,12,18,22,24]$ and quantification $[5,20,21]$, and

*This material is based on research sponsored by NSF under grants CCF-1817242 and CCF-1901098 and by DARPA under the agreement number FA8750-15-2-0087. The U.S. Government is authorized to reproduce and distribute reprints for Governmental purposes notwithstanding any copyright notation thereon. The views and conclusions contained herein are those of the authors and should not be interpreted as necessarily representing the official policies or endorsements, either expressed or implied, of DARPA or the U.S. Government.

Permission to make digital or hard copies of all or part of this work for personal or classroom use is granted without fee provided that copies are not made or distributed for profit or commercial advantage and that copies bear this notice and the full citation on the first page. Copyrights for components of this work owned by others than ACM must be honored. Abstracting with credit is permitted. To copy otherwise, or republish, to post on servers or to redistribute to lists, requires prior specific permission and/or a fee. Request permissions from permissions@acm.org.

ICSE '20 Companion, May 23-29, 2020, Seoul, Republic of Korea

(C) 2020 Association for Computing Machinery

ACM ISBN 978-1-4503-7122-3/20/05 ..\$15.00

https://doi.org/10.1145/3377812.3382174

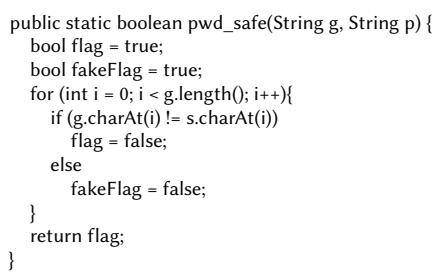

Figure 1: A seemingly timing-channel-free password checking function.

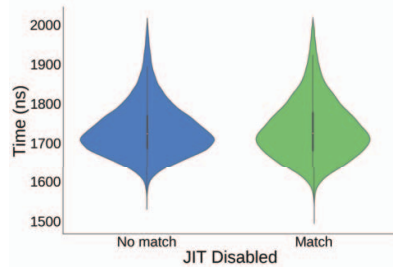

(a) JIT-disabled

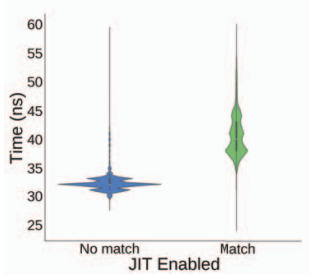

(b) JIT-enabled
Figure 2: Execution time distributions for the password checking function with and without JIT-enabled.

my own work on a static analysis framework for detection of software side-channels called CoCo-CHANNEL [8]) and a constraint caching framework to accelerate side-channel quantification called CASHEW [9].

\section{JIT-INDUCED SIDE CHANNELS}

An underlying assumption common in existing software side-channel analysis techniques $[4,8,12,18]$ is that the source code is the only determiner of the side-channel observable. This is not the case. As I have shown in my research, just-in-time (JIT) compilation, which is crucial to the performance of modern programming languages such as Java and JavaScript, can introduce timing side channels into deceptively secure-looking code fragments when it attempts to optimize paths it deems "hot" [6]. For example, the Java Virtual Machine tracks how often each branch of a conditional branch instruction is taken, and uses this profiling data when optimizing a method to generate native code favoring the more common branch As a result, JIT compilation can introduce timing side channels in cases where the input distribution to the program is non-uniform.

Consider a simple password checking function that compares two strings character by character. If a programmer is concerned with timing vulnerabilities, they would avoid coding a function that returns as soon as there is a mismatch between characters but instead does the same amount of work per loop iteration regardless 


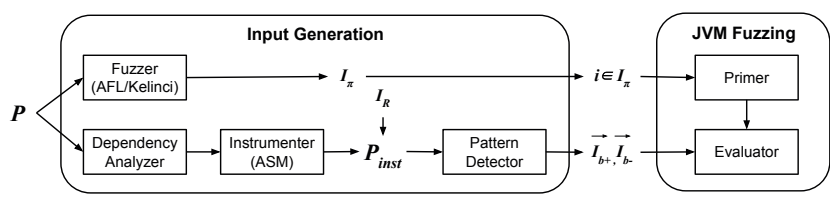

Figure 3: Automated JIT-Induced Side-Channel Detection

of whether the characters match. Figure 1 is such a function. At a static level, this function is devoid of timing side channels. However, once the behavior of the runtime is considered, this is no longer the case. Figure 2a shows two violin plots - the timing distribution of this password checking function when the first characters do not match and the timing distribution when they do. No timing channel is observed - because JIT is disabled. Figure $2 \mathrm{~b}$ shows the analogous violin plots with JIT enabled. A side channel has arisen! This occurs because it is much more likely that the first character of a randomly chosen input does not match that of the password, causing JIT to optimize one path over the other.

JIT-induced side channels could potentially allow an attacker to prime an application in such a way that another user's private data or actions are leaked. I am the first researcher to investigate this class of side-channel vulnerabilities, and my work is the first scientific demonstration of JIT-induced side channels. I have developed three different attack scenarios under which JIT-induced side channels might be leveraged by an attacker and five vulnerability templates to detect susceptible code fragments [6]. Using these templates and attack scenarios, I evaluated three classes from the Java standard library and three JavaScript built-in objects for their vulnerability to JIT-induced side channels, finding many functions vulnerable. I have also induced JITbased side-channels in the Apache Shiro security framework and the GraphHopper route planning server that are large enough in magnitude to be observable over the public internet [6].

\section{AUTOMATIC DETECTION OF JIT-INDUCED SIDE CHANNELS}

The side channels discussed above [6] were induced manually, through intelligent choices of priming input and scenarios. The set of potential priming scenarios for a runtime is extensive, yet not all priming scenarios (or even most) result in observable timing side channels. Therefore, I recently developed an automated process to efficiently and automatically explore the possibility of a timing side channel being induced by the runtime of a program [7].

My automated process consists of two stages, given in Figure 3. First, an Input Generation stage takes a program with some subset of input marked as secret. This phase produces two results. The first is a set of pairs of partitions of the secret domain. Intuitively, these partitions divide the secret domain in disjoint partitions that are most likely to be separable due to a timing side channel. These partitions will be used in the second stage to evaluate if a timing side channel has arisen. The second goal of the input generation phase is to produce a set of priming input. The program under test

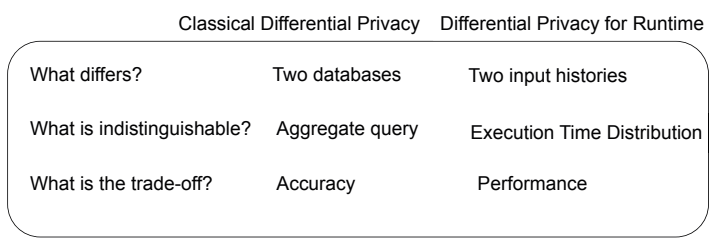

Figure 4: Differential Privacy for Timing Channels

will be primed (i.e. run repeatedly) on these input in an attempt to produce a timing side channel.

The second stage is the fVM Fuzzing stage. The goal of this stage is to answer the question: is there a priming input such that priming in favor of that input results in a timing side channel by which two partitions of the secret domain are distinguishable. The priming input generated in the Input Generation stage are each considered in turn when priming the runtime. Likewise, each pair of partitions of the secret domain generated in the Input Generation are evaluated for separability due to their timing distributions.

Details on the implementation of this process can be found in [7]. I evaluated this technique on three widely used datasets - the Blazer dataset, the Themis dataset, and the DiffFuzz dataset. These datasets have been used in the evaluation of four state-of-theart program analysis tools for side-channel detection $[4,8,12,18]$. Ultimately, I found that many code variants labeled "safe" are in fact vulnerable to JIT-induced timing side channels. My results show that inducing JIT-based side channels can be effectively automated, and also directly contradict the conclusions of four separate side-channel analysis tools. The failure of other program analysis tools in detecting this kind of side channel further motivates the importance of my automated technique.

\section{MITIGATION OF JIT-INDUCED SIDE CHANNELS}

JIT-induced side channels can produce devastating information leaks. It is crucial that the computer science community is not only aware of these vulnerabilities but develops strategies to mitigate their impact. I plan to work towards this end by developing mitigation techniques at the runtime level and leveraging tools from differential privacy to ensure that sensitive user data remains private. Differential privacy allows for the public sharing of aggregate information about a dataset while ensuring that information about any single member of that dataset remains private [14]. I will extend this concept to runtime engines: the runtime state should leverage aggregate information about past input but no one input should be identifiable based on timing information. I will modify the Jikes Research Virtual Machine [3] so that its dynamic compilation mechanism satisfies this constraint. This will defend against the type of JIT-induced side channel present in the Apache Shiro framework and the Graphhopper routing algorithm. However, this mitigation technique will have no chance of being adopted if it introduces significant performance loss. Therefore, I also plan to experimentally evaluate how much execution speed is lost when dynamic compilation is forced to adhere to this differentially private constraint The extension of differential privacy to the new area of JIT-induced side channels is given in Figure 4. 


\section{REFERENCES}

[1] Onur Aciiçmez, Çetin Kaya Koç, and Jean-Pierre Seifert. 2007. On the power of simple branch prediction analysis. In Proceedings of the 2nd ACM symposium on Information, computer and communications security. ACM, 312-320.

[2] Onur Acriçmez, Çetin Kaya Koç, and Jean-Pierre Seifert. 2007. Predicting secret keys via branch prediction. In Cryptographers Track at the RSA Conference. Springer, 225-242.

[3] Bowen Alpern, Steve Augart, Stephen M Blackburn, Maria Butrico, Anthony Cocchi, Perry Cheng, Julian Dolby, Stephen Fink, David Grove, Michael Hind, et al. 2005. The Jikes research virtual machine project: building an open-source research community. IBM Systems fournal 44, 2 (2005), 399-417.

[4] Timos Antonopoulos, Paul Gazzillo, Michael Hicks, Eric Koskinen, Tachio Terauchi, and Shiyi Wei. 2017. Decomposition instead of self-composition for proving the absence of timing channels. In ACM SIGPLAN Notices, Vol. 52. ACM, 362-375.

[5] Lucas Bang, Abdulbaki Aydin, Quoc-Sang Phan, Corina S Păsăreanu, and Tevfik Bultan. 2016. String analysis for side channels with segmented oracles. In Proceedings of the 2016 24th ACM SIGSOFT International Symposium on Foundations of Software Engineering. ACM, 193-204.

[6] Tegan Brennan, Nicolás Rosner, and Tevfik Bultan. 2020. JIT Leaks: Inducing Timing Side Channels through Just-In-Time Compilation. In Proceedings of the 41st IEEE Symposium on Security and Privacy (to appear).

[7] Tegan Brennan, Seemanta Saha, and Tevfik Bultan. 2020. JVM Fuzzing for JITInduced Side-Channel Detection. In Proceedings of the 42nd International Conference on Software Engineering (to appear).

[8] Tegan Brennan, Seemanta Saha, Tevfik Bultan, and Corina S Păsăreanu. 2018 Symbolic path cost analysis for side-channel detection. In Proceedings of the 27th ACM SIGSOFT International Symposium on Software Testing and Analysis. ACM, 27-37.

[9] Tegan Brennan, Nestan Tsiskaridze, Nicolás Rosner, Abdulbaki Aydin, and Tevfik Bultan. 2017. Constraint normalization and parameterized caching for quantitative program analysis. In Proceedings of the 2017 11th Foint Meeting on Foundations of Software Engineering. ACM, 535-546.

[10] Billy Bob Brumley and Risto M Hakala. 2009. Cache-timing template attacks In International Conference on the Theory and Application of Cryptology and Information Security. Springer, 667-684.

[11] Peter Chapman and David Evans. 2011. Automated black-box detection of side-channel vulnerabilities in web applications. In Proceedings of the 18th ACM conference on Computer and communications security. ACM, 263-274.

[12] Jia Chen, Yu Feng, and Isil Dillig. 2017. Precise Detection of Side-Channel Vulnerabilities using Quantitative Cartesian Hoare Logic. In Proceedings of the 2017 ACM SIGSAC Conference on Computer and Communications Security. ACM 875-890

13] Goran Doychev, Boris Köpf, Laurent Mauborgne, and Jan Reineke. 2015. Cacheaudit: A tool for the static analysis of cache side channels. ACM Transactions on Information and System Security (TISSEC) 18, 1 (2015), 4

[14] Cynthia Dwork. 2011. Differential privacy. Encyclopedia of Cryptography and Security (2011), 338-340.

[15] Paul Kocher, Daniel Genkin, Daniel Gruss, Werner Haas, Mike Hamburg, Moritz Lipp, Stefan Mangard, Thomas Prescher, Michael Schwarz, and Yuval Yarom. 2018. Spectre attacks: Exploiting speculative execution. arXiv preprint arXiv:1801.01203 (2018)

[16] Moritz Lipp, Michael Schwarz, Daniel Gruss, Thomas Prescher, Werner Haas, Stefan Mangard, Paul Kocher, Daniel Genkin, Yuval Yarom, and Mike Hamburg. 2018. Meltdown. arXiv preprint arXiv:1801.01207 (2018)

[17] Fangfei Liu, Yuval Yarom, Oian Ge, Gernot Heiser, and Ruby B Lee. 2015. Lastlevel cache side-channel attacks are practical. In Security and Privacy (SP), 2015 IEEE Symposium on. IEEE, 605-622.

[18] Shirin Nilizadeh, Yannic Noller, and Corina S Pasareanu. 2018. DifFuzz: Differential Fuzzing for Side-Channel Analysis. arXiv preprint arXiv:1811.07005 (2018).

[19] Dag Arne Osvik, Adi Shamir, and Eran Tromer. 2006. Cache attacks and countermeasures: the case of AES. In Cryptographers' Track at the RSA Conference. Springer, 1-20.

[20] Corina S Pasareanu, Quoc-Sang Phan, and Pasquale Malacaria. 2016. Multi-run side-channel analysis using Symbolic Execution and Max-SMT. In Computer Security Foundations Symposium (CSF), 2016 IEEE 29th. IEEE, 387-400.

[21] Ouoc-Sang Phan, Lucas Bang, Corina S Pasareanu, Pasquale Malacaria, and Tevfik Bultan. 2017. Synthesis of Adaptive Side-Channel Attacks. In Computer Security Foundations Symposium (CSF), 2017 IEEE 30th. IEEE, 328-342.

[22] Nicolás Rosner, Ismet Burak Kadron, Lucas Bang, and Tevfik Bultan. 2018. Profit Detecting and Quantifying Side Channels in Networked Applications.

[23] Yuval Yarom and Katrina Falkner. 2014. FLUSH+ RELOAD: A High Resolution, Low Noise, L3 Cache Side-Channel Attack.. In USENIX Security Symposium. 719 732

[24] Kehuan Zhang, Zhou Li, Rui Wang, XiaoFeng Wang, and Shuo Chen. 2010. Sidebuster: automated detection and quantification of side-channel leaks in web application development. In Proceedings of the 17th ACM conference on Computer 